\title{
SEARCH FOR B-DECAY TO HIGGS BOSONS FOR HIGGS BOSON MASSES BETWEEN 50 AND $210 \mathrm{MeV} / \mathrm{c}^{2} \leftarrow$
}

\author{
A. SNYDER ${ }^{\text {a }}$, W.N. MURRAY ${ }^{\text {a }}$, G. ABRAMS ${ }^{\text {b }}$, C.E. ADOLPHSEN ${ }^{\text {c }}$, C. AKERLOF ${ }^{\text {d }}$, \\ J.P. ALEXANDER ${ }^{e, 1}$, M. ALVAREZ ${ }^{f, 2}$, D. AMIDEI ${ }^{b, 3}$, A.R. BADEN ${ }^{b, 4}$, B.C. BARISH $^{g}$, \\ T. BARKLOW ${ }^{\text {e }}$, B.A. BARNETT ${ }^{\text {h }}$, J. BARTELT ${ }^{\text {e }}$, D. BLOCKUS ${ }^{\text {a }}$, G. BONVICINI $^{d}$, \\ A. BOYARSKI ${ }^{\text {e }}$, J. BOYER ${ }^{\text {b }}$, B. BRABSON ${ }^{a}$, A. BREAKSTONE ${ }^{i}$, J.M. BROM $^{\mathrm{a}, 5}$, F. BULOS $^{\text {e }}$, \\ P.R. BURCHAT ${ }^{c}$, D.L. BURKE ${ }^{\mathrm{e}}$, F. BUTLER $^{\mathrm{b}, 6}$, F. CALVINO $^{\mathrm{f}, 7}$, R.J. CENCE $^{\mathrm{i}}$, J. CHAPMAN ${ }^{\mathrm{d}}$, \\ D. CORDS $^{\mathrm{e}}$, D.P. COUPAL ${ }^{\mathrm{e}}$, H.C. DESTAEBLER ${ }^{\mathrm{e}}$, D.E. DORFAN ${ }^{\mathrm{c}}$, J.M. DORFAN $^{\mathrm{e}}$, \\ P.S. DRELL ${ }^{b, 1}$, G.J. FELDMAN ${ }^{\mathrm{e}}$, E. FERNANDEZ ${ }^{\text {f, }}$, R.C. FIELD ${ }^{\mathrm{e}}$, W.T. FORD ${ }^{\mathrm{f}}$, C. FORDHAM ${ }^{\mathrm{e}}$,

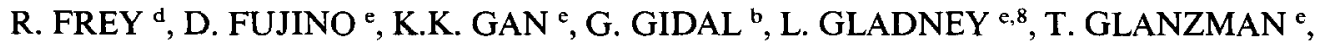 \\ M.S. GOLD ${ }^{b}$, G. GOLDHABER ${ }^{b}$, P. GROSSE-WIESMANN ${ }^{\mathrm{c}}$, J. HAGGERTY $^{\mathrm{b}, 9}$, G. HANSON $^{\mathrm{e}}$, \\ R. HARR ${ }^{b}$, F.A. HARRIS ${ }^{c}$, C.M. HAWKES ${ }^{\mathrm{g}}$, K. HAYES ${ }^{\mathrm{e}}$, D. HERRUP ${ }^{\mathrm{b}}{ }^{\mathrm{b}}{ }^{10}$, C.A. HEUSCH $^{\mathrm{c}}$, \\ T. HIMEL ${ }^{\mathrm{e}}$, R.J. HOLLEBEEK ${ }^{\mathrm{e}, 8}$, D. HUTCHINSON ${ }^{\mathrm{e}}$, J. HYLEN ${ }^{\text {h }}$, W.R. INNES ${ }^{\mathrm{e}}$, M. JAFFRE $^{\mathrm{b}, 11}$, \\ J.A. JAROS ${ }^{\text {e }}$, I. JURICIC ${ }^{\text {b, } 12}$, J.A. KADYK ${ }^{\text {b }}$, D. KARLEN ${ }^{\text {e, }}{ }^{13}$, J. KENT $^{c}$, S.R. KLEIN ${ }^{e, 14}$, \\ W. KOSKA ${ }^{\text {d }}$, W. KOZANECKI ${ }^{\mathrm{e}}$, A.J. LANKFORD ${ }^{\mathrm{e}}$, R.R. LARSEN ${ }^{\mathrm{c}}$, B.W. LECLAIRE ${ }^{\mathrm{c}, 15}$, \\ M.E. LEVI ${ }^{b}$, A.M. LITKE ${ }^{c}$, N.S. LOCKYER ${ }^{e, 8}$, V. LÜTH ${ }^{e}$, J.A.J. MATTHEWS ${ }^{\text {h }}$, D.I. MEYER ${ }^{d}$, \\ B.D. MILLIKEN ${ }^{8}$, K.C. MOFFEIT e ${ }^{\text {, L. MÜLLER }}{ }^{\mathrm{e}, 16}$, J. NASH ${ }^{\mathrm{e}}$, M.E. NELSON $^{\mathrm{g}}$, D. NITZ $^{\mathrm{d}}$, \\ H. OGREN ${ }^{\text {a }}$, R.A. ONG ${ }^{\text {e } 3}$, K.F. O'SHAUGHNESSY ${ }^{\text {e }}$, S.I. PARKER ${ }^{i}$, C. PECK ${ }^{\text {g }}$, M.L. PERL ${ }^{\mathrm{e}}$, \\ A. PETERSEN ${ }^{\mathrm{e}, 17}$, M. PETRADZA ${ }^{\text {d }}$, F.C. PORTER ${ }^{\mathrm{g}}$, P. RANKIN $^{\mathrm{f}}$, B. RICHTER ${ }^{\mathrm{c}}$, K. RILES $^{\mathrm{c}}$, \\ P.C. ROWSON ${ }^{b, 12}$, D.R. RUST ${ }^{a}$, H.F.W. SADROZINSKI ${ }^{c}$, T. SCHAAD $^{\mathrm{j}, 18}$, T.L. SCHALK ${ }^{\mathrm{c}}$, \\ H. SCHELLMAN ${ }^{b, 10}$, W.B. SCHMIDKE ${ }^{b}$, A.S. SCHWARZ ${ }^{c, 19}$, A. SEIDEN $^{c}$, P.D. SHELDON ${ }^{b, 20}$, \\ J.G. SMITH ${ }^{\text {f }}$, E. SODERSTROM ${ }^{\mathrm{g}}$, D.P. STOKER ${ }^{\text {h }}$, R. STROYNOWSKI ${ }^{\mathrm{g}}$, R. THUN ${ }^{\mathrm{d}}$, \\ G.H. TRILLING ${ }^{\mathrm{b}}, \mathbf{R}$. TSCHIRHART ${ }^{\mathrm{d}, 21}$, R. VAN KOOTEN $^{\mathrm{e}}$, H. VELTMAN $^{\mathrm{d}, 22}{ }^{\mathrm{d}}$, \\ P. VORUGANTI ${ }^{e}$, S.R. WAGNER ${ }^{\text {f }}$, P. WEBER ${ }^{\mathrm{f}}$, A.J. WEINSTEIN ${ }^{\mathrm{c}}$, S. WEISZ $^{\mathrm{c}, 23}$, S.L. WHITE $^{\mathrm{f}, 24}$ \\ E. WICKLUND ${ }^{\text {g }}$, A.J. WEIR ${ }^{\text {g }}$, D.R. WOOD ${ }^{\mathrm{b}, 23}$, D.Y. WU ${ }^{\mathrm{g}}$ and J.M. YELTON ${ }^{\mathrm{e}, 25}$ \\ a Indiana University, Bloomington, IN 47405, USA \\ b Lawrence Berkeley Laboratory and Department of Physics, University of California, Berkeley, CA 94720, USA \\ c University of California, Santa Cruz, CA 95064, USA \\ d University of Michigan, Ann Arbor, MI 48109, USA \\ - Stanford Linear Accelerator Center, Stanford University, Stanford, CA 94309, USA. \\ - University of Colorado, Boulder, CO 80309, USA \\ \& California Institute of Technology, Pasadena, CA 91125, USA \\ h Johns Hopkins University, Baltimore, MD 21218, USA \\ i University of Hawaii, Honolulu, HI 96822, USA \\ j Harvard University, Cambridge, MA 02138, USA
}

Received 11 July 1989

\footnotetext{
We use data from the Mark II experiment at PEP to search for the process $\mathrm{B} \rightarrow \mathrm{h}^{0} \mathrm{X}$ for $m_{\mathrm{h}^{0}}$ between 50 and $210 \mathrm{MeV} / c^{2}$. No evidence for the Higgs boson is seen in this mass range. The limit obtained rules out the standard Higgs boson for masses between 70 and $210 \mathrm{MeV} / \mathrm{c}^{2}$ and significantly constrains extensions of the Higgs sector.
} 
Recently several authors [1] have pointed out a gap in the exclusion of the standard Higgs boson for Higgs boson masses less than $2 m_{\mu}$ where $\mathrm{e}^{+} \mathrm{e}^{-}$is the

* This work was supported in part by Department of Energy contracts DE-AC03-81ER40050 (CIT), DE-AM0376SF00010 (UCSC), DE-AC02-86ER40253 (Colorado), DEAC03-83ER40103 (Hawaii), DE-AC02-84ER40125 (Indiana), DE-AC03-76SF00098 (LBL), DE-AC02-84ER40125 (Michigan), and DE-AC03-76SF00515 (SLAC), and by the National Science Foundation (Johns Hopkins).

1 Present address: Cornell University, Ithaca, NY 14853, USA.

2 Present address: Universidad Autónoma de Barcelona, Bellaterra, Spain.

${ }^{3}$ Present address: University of Chicago, Chicago, IL 60637, USA.

${ }^{4}$ Present address: Harvard University, Cambridge, MA 02138, USA.

5 Present address: Centre de Recherches Nucléaires, F-67037 Strasbourg, France.

6 Present address: University of Oklahoma, Norman, OK 73019, USA.

${ }^{7}$ Present address: Universitat Politecnica de Catalunya, ETSEIB-DEN Barcelona, Spain.

${ }^{8}$ Present address: University of Pennsylvania, Philadelphia, PA 19104, USA.

9 Present address: Brookhaven National Laboratory, Upton, NY 11973, USA.

10 Present address: Fermilab, Batavia, IL 60510, USA.

1 Present address: Laboratoire de l'Accélérateur Linéaire, F91405 Orsay, France.

12 Present address: Columbia University, New York, NY 10027, USA.

${ }^{13}$ Present address: Carleton University, Ottawa, Ontario, Canada K1S 5B6.

14 Present address: Boston University, Boston, MA 02215, USA.

15 Present address: University of Wisconsin, Madison, WI 53706, USA.

16 Present address: Laboratorium für Hochenergie Physik Bern, CH-3012 Bern, Switzerland.

17 Present address: SCS, Hamburg, FRG.

18 Present address: Université de Genève $\mathrm{CH}-1211$ Geneva 4, Switzerland.

19 Present address: Max-Planck Institut, D-8000 Munich, FRG.

20 Present address: University of Illinois, Urbana, IL 61801, USA.

21 Present address: Princeton University, Physics Department, Princeton, NJ 08544, USA.

22 Present address: University of California, Berkeley, CA 94720 , USA.

23 Present address: CERN, CH-1211 Geneva 23, Switzerland.

${ }^{24}$ Present address: University of Tennessee, Knoxville, TN 37996 USA.

25 Present address: University of Florida, Gainsville, F1 3261 I, USA.

${ }^{26}$ Present address: Therma-Wave Corporation, Fremont, CA 94539 , USA. dominant decay mode. The $7 \mathrm{GeV} / c^{2}$ theoretical lower bound on the Higgs boson mass established by Linde and Weinberg [2] disappears for $m_{\mathrm{t}} \geqslant 80 \mathrm{GeV} /$ $c^{2}$ and the significance of the experimental limits for Higgs boson production in K-decay [ 3 ] is obscured by theoretical uncertainties associated with bound state effects. The $r$ - and $\psi$-decay experiments have not yet penetrated this mass region where a large QED background $\left(\mathrm{e}^{+} \mathrm{e}^{-} \gamma\right)$ can be expected.

B-decay can fill this gap. The predicted rate for Higgs boson production in B-decay is large [4] and the theoretical uncertainties are relatively mild. A controversy about the large predicted rate has been resolved by an alternate derivation of the result [5].

In this paper, using data from the Mark II detector at PEP, we present the results of a search for the decay of B-hadrons to Higgs boson, where the Higgs boson decays to $\mathrm{e}^{+} \mathrm{e}^{-}$. The mass region from 50 to 210 $\mathrm{MeV} / \mathrm{c}^{2}$ is examined. The search was based on data taken with the Mark II detector in both the PEP5 and Upgrade configurations. The detectors have been described in detail elsewhere [6].

The branching fraction for the decay $\mathrm{B} \rightarrow \mathrm{h}^{0} \mathrm{X}$ in the standard model (for small Higgs boson masses) is given by

$B\left(\mathbf{B} \rightarrow \mathbf{h}^{0} \mathrm{X}\right)=\frac{K \times 10^{-6} \times\left(m_{\mathrm{t}} / m_{\mathrm{b}}\right)^{4}}{1+K \times 10^{-6} \times\left(m_{\mathrm{t}} / m_{\mathrm{b}}\right)^{4}}$,

where $K$ is calculated to be between 4 and 6 . Using $K=4, m_{\mathrm{b}}=4.9 \mathrm{GeV} / c^{2}$ and $m_{1}=80 \mathrm{GeV} / c^{2}$ (the value for which the theoretical lower bound on the Higgs boson mass disappears $), B\left(B \rightarrow h^{0} \mathrm{X}\right)=0.22$. This high rate implies that modest limits can exclude the standard Higgs boson. However, conclusions based on analyses not specifically aimed at the Higgs boson (e.g. neutral current limits [7]) may not be valid. These analyses typically select electrons consistent with production at the primary vertex and may not be sensitive to low mass Higgs boson production since the Higgs boson lifetime would be significantly different from zero for masses below $2 m_{\mu}$.

We employ three striking features of Higgs boson production in B-decays to discriminate it from background:

(1) At the quark level, the decay $B \rightarrow h^{0} X$ proceeds through the two body process $b \rightarrow h^{0}$ s. In the spectator model, this leads to a very hard Higgs boson momentum spectrum in the B-meson center-of-mass. 
(2) The B-hadron fragmentation function is also very hard. On the average, a B-hadron produced in $\mathrm{e}^{+} \mathrm{e}^{-}$collisions carries $80 \%$ of the beam energy. When combined with (1), this leads to a stiff momentum spectrum for the Higgs boson. For example, a Higgs boson produced at $90^{\circ}$ with respect to the $B$ line-offlight in the $B$ rest frame carries about half the energy of the B-hadron. Fig. 1 shows a scatter plot of $P_{1}$ versus $P_{\|}$(defined relative to the thrust direction) for a sample of Monte Carlo generated Higgs boson decays. A simple spectator model is used to model the decay $\mathrm{B} \rightarrow \mathrm{h}^{0} \mathrm{X}$. Version 6.3 of the LUND Monte Carlo with Peterson fragmentation [8] is used to model the b-quark fragmentation process. Based on this plot we use $P_{\|}>3.0 \mathrm{GeV} / c$ and $P_{\mathrm{t}}>1.0 \mathrm{GeV} / c$ to select Higgs boson decays of B-hadrons. These cuts accept $\approx 70 \%$ of the $\mathrm{h}^{0} \rightarrow \mathrm{e}^{+} \mathrm{e}^{-}$decays reconstructed by the detector.

(3) The lifetime of a Higgs boson in this mass range would be relatively long. It is given by [9]

$$
1 / \tau=\Gamma\left(\mathrm{h}^{0} \rightarrow \mathrm{e}^{+} \mathrm{e}^{-}\right)=G_{\mathrm{F}} m_{\mathrm{e}}^{2} m_{\mathrm{h}} / 4 \pi \sqrt{2}
$$

for $m_{\mathrm{e}} \ll m_{\mathrm{h}}$. For a $200 \mathrm{MeV} / c^{2}$ Higgs boson a $c \tau$ of $3 \mathrm{~mm}$ is expected. This leads to a typical decay length in the lab $(\gamma \beta c \tau)$ of $\approx 7 \mathrm{~cm}$ for $200 \mathrm{MeV} / c^{2}$ Higgs bosons from the decay of $\mathrm{B}$-hadrons produced in $\mathrm{e}^{+} \mathrm{e}^{-}$ collisions at $29 \mathrm{GeV} / c^{2}$. We also cut on $R$, the transverse distance from the beamline of vertices of candidate $\mathrm{e}^{+} \mathrm{e}^{-}$pairs. To eliminate background from $\mathrm{B}-$ or D-meson semi-leptonic decays, we require $R$ to be greater than $1 \mathrm{~cm}$.

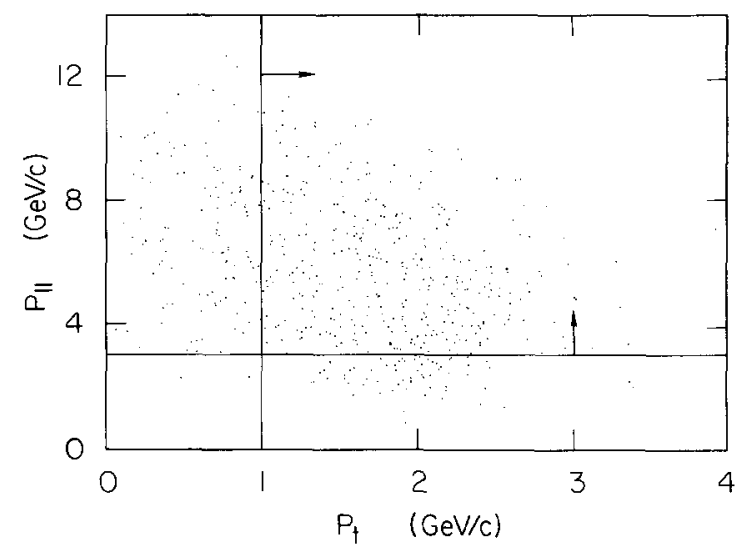

Fig. 1. $P_{\mathrm{t}}$ versus $P_{\mathrm{k}}$. The lines indicate the cuts at $P_{\mathrm{t}}>1.0 \mathrm{GeV} / \mathrm{C}$ and $P_{\|}>3.0 \mathrm{GeV} / c$. These events were generated with $m_{\mathrm{h} 0}=200$ $\mathrm{MeV} / \mathrm{c}^{2}$.
The lifetime of the Higgs boson, see eq. (2), is inversely proportional to its mass. The typical value for $\gamma$ also increases as $1 / m_{\mathrm{h}}$ so that the decay length in the lab increases as $1 / m_{\mathrm{h}}^{2}$. For a $50 \mathrm{MeV} / c^{2}$ Higgs boson, the expected decay length is $120 \mathrm{~cm}$. This tends to reduce our Higgs boson detection efficiency at low Higgs boson mass (see fig. 2a). Because of this deterioration in the efficiency, as well as background due to $\gamma$ conversions, we do not attempt to search for Higgs bosons with masses less than $50 \mathrm{MeV} / c^{2}$.

We applied the cuts defined above to hadronic events selected from both the PEP5 $\left(209 \mathrm{pb}^{-1}\right)$ and Upgrade (28 $\mathrm{pb}^{-1}$ ) running of the Mark II. Hadronic events were selected with the following cuts: (1) at least 5 reconstructed charged tracks must be found
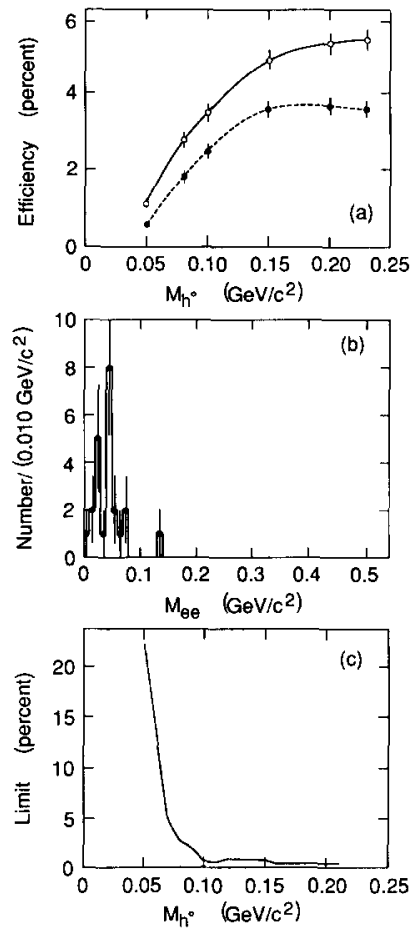

Fig. 2. (a) Higgs boson detection efficiency as a function of mass $\left(m_{\mathrm{h}^{0}}\right.$ ) for $P_{\mathrm{1}}>1.0 \mathrm{GeV} / c, P_{\|}>3.0 \mathrm{GeV} / c$ and $R>1 \mathrm{~cm}$. The upper curve gives the full efficiency regardless of the reconstructed mass. The lower (dashed) curve indicates the efficiency for finding the reconstructed mass within $20 \mathrm{MeV} / \mathrm{c}^{2}$ of the actual mass

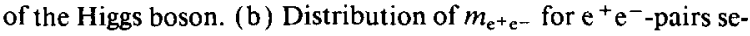
lected by $P_{1}>1.0 \mathrm{GeV} / c, P_{\|}>3.0 \mathrm{GeV} / c$, and $R>1 \mathrm{~cm}$. (c) Limit on $B\left(\mathrm{~B} \rightarrow \mathrm{h}^{0} \mathrm{X}\right) \times B\left(\mathrm{~h}^{0} \rightarrow \mathrm{e}^{+} \mathrm{e}^{-}\right)$as a function of Higgs boson mass $\left(m_{\mathrm{h}^{0}}\right)$. 
coming from within $4 \mathrm{~cm}$ radially and $7 \mathrm{~cm}$ axially of the expected interaction point; (2) the scalar sum of the charged track momenta had to be at least 7.25 $\mathrm{GeV} / c$; (3) the sum of the visible charged and neutral energy had to be at least $7.5 \mathrm{GeV}$.

Fig. 2b shows the $\mathrm{e}^{+} \mathrm{e}^{-}$mass distribution for the selected events. There are a few events at low mass. These are consistent with $\gamma$-conversions in the beam pipe and vertex chamber walls. Monte Carlo studies indicate that the dominant sources of these high momentum photons are initial state radiation and neutral pions in B-decays. At high masses, Dalitz decays can cause some background. There is no evidence for a Higgs boson. For purposes of setting a limit on Higgs boson production we make the conservative assumption that all the $\mathrm{e}^{+} \mathrm{e}^{-}$-pairs observed are "signal". We look at the data in $40 \mathrm{MeV} / \mathrm{c}^{2}$ bins and use Poisson statistics to set a $90 \%$ confidence level limit on the number of Higgs bosons with a mass in the middle of the mass bin. Using the efficiency for reconstructing a Higgs boson within $\pm 20 \mathrm{MeV} / \mathrm{c}^{2}$ of its true mass (see the dashed curve in fig. $2 a$ ) we obtain a limit on the number of Higgs bosons produced. An additional factor of 0.95 is included in the efficiency to account for reduced track finding efficiency during running periods when the drift chamber could not be run at full voltage [10].

The number of B-hadrons produced can be obtained from the fact that $\mathrm{e}^{+} \mathrm{e}^{-} \rightarrow \mathrm{b} \overline{\mathrm{b}}$ events comprise $9 \%$ of all $\mathrm{e}^{+} \mathrm{e}^{-}$hadronic final states and from the luminosity of $237 \mathrm{pb}^{-1}$ obtained in the PEP5 and Upgrade running of the Mark II experiment. This yields $\approx 18000$ for the estimated number of produced $\mathrm{B}$ hadrons. We increase our limit by $15 \%$ to account for any systematic error in the efficiency determination. Details of the efficiency determination are given below. The resulting limit at $90 \%$ confidence level on the product branching fraction $B\left(\mathrm{~B} \rightarrow \mathrm{h}^{0} \mathrm{X}\right)$ $\times B\left(\mathrm{~h}^{0} \rightarrow \mathrm{e}^{+} \mathrm{e}^{-}\right)$is displayed in fig. 2c. The decay mode $\mathrm{h}^{0} \rightarrow \mathrm{e}^{+} \mathrm{e}^{-}$is expected to dominate Higgs boson decay for the mass range under consideration (see ref. [9]).

The efficiency is estimated by generating $B \bar{B}$ events where the B-meson is forced to decay to a Higgs boson. Samples of $\approx 6600$ events were generated using the spectator model to simulate the $\mathrm{B} \rightarrow \mathrm{h}^{0} \mathrm{X}$ decay process. This was done for Higgs boson masses of 50 , $80,100,150,200$ and $230 \mathrm{MeV} / c^{2}$. These events were subjected to the standard Mark II analysis package and to the same cuts applied to the data. In addition, for electron identification, fiducial cuts were used to simulate cracks in the liquid argon calorimeter [11]. The fiducial cuts constrained the intersection of the tracks with the calorimeter to be less than $1.75 \mathrm{~m}$ longitudinally distant from the interaction point, and required that no track enter the calorimeter within 0.04 radians of an azimuthal crack between calorimeter octants. Shower simulation was done by the mark II fast shower look-up program (OVEREZY). The results are shown in fig. $2 a$. The smooth curves connecting the points are used to extrapolate to masses between the generated masses. The lower dashed curve shows the efficiency for finding the Higgs boson within $\pm 20 \mathrm{MeV} / \mathrm{c}^{2}$ of its actual mass.

The significant contributions to the systematic error are given in table 1 . These errors were evaluated by varying the parameters of the Monte Carlo simulation and looking for the effects on the estimated efficiency. They may well be over-estimates since in most cases the results were consistent with the nominal parameter settings within the statistical error of the Monte Carlo samples. We have used PEP5 detector simulation for all our efficiency estimates. The Upgrade, with its higher field, multi-hit electronics and larger number of wires in the central drift chamber should have substantially better efficiency. Thus, the efficiency used in obtaining the limit is underestimated. Nonetheless, to be conservative, as mentioned before, we have increased our limit by $15 \%$ to account for systematics. A brief description of each of the sources of systematic error follows.

The Higgs boson lifetime $(c \tau)$ is uncertain near $\mu \mu$ threshold where the $\mathrm{h}^{0} \rightarrow \gamma \gamma$ rate can become comparable to $\mathrm{h}^{0} \rightarrow \mathrm{e}^{+} \mathrm{e}^{-}$( see ref. [9]). We have checked the efficiency with Monte Carlo simulations using

Table 1

Estimated contributions to systematic error on the efficiency for detecting the electron pair from Higgs boson decay. The lifetime uncertainty only applies in the neighborhood of $200 \mathrm{MeV} / \mathrm{c}^{2}$.

\begin{tabular}{lll}
\hline Source of error & $\begin{array}{l}\text { Estimate } \\
\text { contribution }\end{array}$ & Estimation method \\
\hline spectator model & $\pm 5 \%$ & model variation \\
fragmentation & $\pm 5 \%$ & model variation \\
Higgs lifetime & $\pm 5 \%$ & variation of lifetime \\
Monte Carlo statistics & $\pm(7-10) \%$ & Monte Carlo \\
\hline
\end{tabular}


lifetimes as low as $\frac{1}{2}$ the lifetime expected without the $\gamma \gamma$-mode. The efficiency change found is less than the $5 \%$ statistical error. As a further check on the extreme case of a zero lifetime Higgs boson, we have repeated the analysis with no cut on the radius of the vertex. This re-analysis picks up 2 events within $\pm 20 \mathrm{MeV} /$ $c^{2}$ of $200 \mathrm{MeV} / c^{2}$. Even this extreme case still yields a $90 \%$ confidence level limit of $\approx 1 \%$ on $B\left(B \rightarrow h^{0} \mathrm{X}\right)$ $\times B\left(\mathrm{~h}^{0} \rightarrow \mathrm{e}^{+} \mathrm{e}^{-}\right)$.

Systematic errors in the fragmentation model were estimated by generating event samples with $m_{\mathrm{h} 0}=150$ and $200 \mathrm{MeV} / \mathrm{c}^{2}$ with the Peterson fragmentation parameter $\left(\epsilon_{b}\right)$ varied by $\pm 40 \%$. This corresponds to varying the mean value of the b-quark fragmentation function by the experimental error established by Ong et al. [12]. To estimate the effect of uncertainty of the b-quark mass in the spectator model we scaled the generated Higgs boson momentum down by $10 \%$. This corresponds to changing the b-quark mass from the LUND default value of $5.0 \mathrm{GeV} / c^{2}$ to $\approx 4.5 \mathrm{GeV} /$ $c^{2}$.

Our limit is more than a factor of 4 below the $22 \%$ branching fraction expected for a standard Higgs boson with a mass in the 70 to $210 \mathrm{MeV} / \mathrm{c}^{2}$ mass range. For the masses above $100 \mathrm{MeV} / c^{2}$, the limit is a factor of 20 or more below the predicted branching fraction. This excludes the standard Higgs boson for any reasonable variation in the theoretical predictions. For masses near the lower limit of our search $(50$ $\mathrm{MeV} / \mathrm{c}^{2}$ ), a combination of background and poor efficiency make the limit obtained relatively poor. While a $22 \% \mathrm{~B} \rightarrow \mathrm{h}^{0} \mathrm{X}$ branching fraction is formally excluded (at $90 \%$ confidence level) the conclusion is not as secure as in the higher mass regime.

Our result can also constrain extended Higgs models. The simplest extension is to have two Higgs doublets [13]. Since the lifetime of the Higgs boson may vary from the single Higgs doublet prediction when the Higgs sector is increased, we have repeated our analysis for a variety of lifetimes for the cases of 100,150 and $200 \mathrm{MeV} / \mathrm{c}^{2}$ Higgs boson masses. The results are given in table 2 . The decay length cut is removed for the zero lifetime case. This results in a few more background events being accepted. Similar limits apply to intermediate masses. The results in table 2 can be compared with a branching fraction of $\approx 5 \%$ for $\mathrm{B} \rightarrow \mathrm{h}^{0} \mathrm{X}$ that is expected for a two doublet Higgs if $m_{\mathrm{t}}$ is $50 \mathrm{GeV} / c^{2}$ and if the vacuum expecta-
Table 2

Upper limit on $B\left(\mathrm{~B} \rightarrow \mathrm{h}^{0} \mathrm{X}\right) \times B\left(\mathrm{~h}^{0} \rightarrow \mathrm{e}^{+} \mathrm{e}^{-}\right)$for various values of the Higgs boson mass and lifetime.

\begin{tabular}{llll}
\hline $\begin{array}{l}\text { Lifetime } \\
(c \tau)(\mathrm{mm})\end{array}$ & $\begin{array}{l}100 \\
\mathrm{MeV} / \mathrm{c}^{2}\end{array}$ & $\begin{array}{l}150 \\
\mathrm{MeV} / \mathrm{c}^{2}\end{array}$ & $\begin{array}{l}200 \\
\mathrm{MeV} / \mathrm{c}^{2}\end{array}$ \\
\hline 0 & $1.0 \%$ & $0.72 \%$ & $0.71 \%$ \\
3 & $0.49 \%$ & $0.66 \%$ & $0.45 \%$ \\
6 & $0.73 \%$ & $0.87 \%$ & $0.46 \%$ \\
12 & $1.1 \%$ & $1.3 \%$ & $0.59 \%$ \\
24 & $2.4 \%$ & $2.0 \%$ & $1.3 \%$ \\
\hline
\end{tabular}

tion values of the two doublets are equal [14]. We conclude that if there is a non-standard Higgs boson between 100 and $210 \mathrm{MeV} / \mathrm{c}^{2}$ either the $\mathrm{B}$ to Higgs boson decay rate is suppressed or that the lifetime is long. This would imply that the ratio of the vacuum expectation values for the two Higgs doublets is substantially different from one.

We thank Howard E. Haber for useful discussions that materially aided in the preparation of this paper. We are also grateful for the efforts of the PEP staff, whose excellent work has made this paper possible.

\section{References}

[1] S. Dawson, Limits on light Higgs bosons, in: Proc. 1988 DPF Meeting (Storrs. CT, August 1988), preprint BNL-99-0913; S. Raby, G.B. West and C.M. Hoffman, Are there really any experimental limits on a light Higgs boson?, preprint LAUR-88-2667;

J.F. Gunion, An overview of discovery techniques and excluded mass regions for the standard Higgs boson, in: Proc. XXIV Intern. Conf. on High energy physics (Munich, August 1988), preprint UCD-88-3.

[2] A.D. Linde JETP Lett. 23 (1964);

S. Weinberg, Phys. Rev. Lett. 36 (1976) 294.

[3] T. Yamazaki et al., Phys. Rev. Lett. 52 (1984) 1089; N. Baker et al., Phys. Rev. Lett. 59 (1987) 2832; a comprehensive review can be found in J.F. Gunion and H.E. Haber, The physics of Higgs bosons: the Higgs hunter's guide, Phys. Rep., to be published.

[4] R.S. Willey and H.L. Yu, Phys. Rev. D 26 (1982) 3086; H.E. Haber, A.S. Schwarz and A.E. Snyder, Nucl. Phys. B 294 (1987) 301.

[5] B. Grinstein, L. Hall and L. Randall, Phys. Lett. B 211 (1989) 363.

[6] The PEP5 detector is described in R.H. Schindler et al., Phys. Rev. D 24 (1981) 78; the Upgrade detector is described in S. Wagner et al., Phys. Rev. D 35 (1987) 2850, and references therein. 
[7] CLEO Collab, P. Haas et al., Phys. Rev. Lett. 60 (1988) 1614.

[8] T. Sjostrand, Comput. Phys. Commun. 39 (1986) 347; B. Anderson, G. Gustafson and C. Peterson, Phys. Scr. 19 (1979) 184;

Mark II Collab., A. Peterson et al., Phys. Rev. D 37 (1988) 1.

[9] J. Ellis, M.K. Gaillard and D.V. Nanopoulos, Nucl. Phys. B 106 (1976) 292.

[10] S. Klein, Ph.D. Thesis, Stanford University, SLAC-Report330 (1988);

H. Schellman, Ph.D. Thesis, Lawrence Berkeley Laboratory Report LBL-18699 (1984);

TG Technical Note 247 (1983).
[11 ] D.A. Karlen, Ph.D. Thesis, Stanford University, SLACReport-325 (1988).

[12] MARK II Callab. Ong et al., Phys. Rev. Lett. 60 (1988) 2587.

[13] J.F. Gunion and H.E. Haber, Higgs boson beyond the standard model, in: Proc. XXIV Intern. Conf. on High energy physics (Munich, August 1988), preprint UCD-8836.

[14] L.J. Hall and M.B. Wise, Nucl. Phys. B 187 (1981) 397. 